\title{
Correction to: Optimal Location for Renewable Gas Production and Distribution Facilities: Resource Planning and Management
}

\author{
Bhaba R. Sarker ${ }^{1} \cdot$ Bingqing Wu$^{1} \cdot$ Krishna P Paudel $^{2}$
}

Published online: 18 August 2021

๑) Springer Science+Business Media, LLC, part of Springer Nature 2021

\author{
Correction to: BioEnergy Research \\ https://doi.org/10.1007/s12155-021-10278-0
}

In the "A Numerical Illustration" section, the mathematical expression in matrix format were mistakenly presented. The correct form are as follows:

\subsection{A numerical illustration}

Assume a small region with four zip codes grows three kinds of residues. For simplicity, let the fixed building cost $\mathrm{C}^{\mathrm{f}}$ and variable building $\cos t \mathrm{C}_{\mathrm{n}}^{\mathrm{v}}$ be 0 each because they are just constants in the model. For ease of computation, let us assume that two anaerobic reactors and two condensers are to be located so that the liquefied SYNGAS are delivered in three locations in the region. The information are listed as follows: total number of types of residues, $K=3$; total number of zip codes in the region, $Z=4$; total number of reactors to be built, $N=2$; total number of condensers to be built, $M$ $=2$; total number of delivery points, $L=3$.

Let the availability of residue $k$ in the hub in zip code $z$ be $\mathbf{A}=\left[A_{z k}\right]=\left[\begin{array}{lll}3.4 & 2.5 & 2.2 \\ 2.3 & 4.5 & 3.3 \\ 2.7 & 3.6 & 2.3 \\ 4.7 & 5.0 & 4.9\end{array}\right]$ (truck-load) and the unit cost

The original article can be found online at https://doi.org/10.1007/ s12155-021-10278-0

Bhaba R. Sarker

bsarker@1su.edu

Bingqing Wu

bingqingwu2015@gmail.com

Krishna P Paudel

kpaude1@agcenter.lsu.edu

1 Department of Mechanical and Industrial Engineering, Louisiana State University, Baton Rouge, LA 70803, USA

2 Department of Agricultural Economics \& Agribusiness, LSU and LSU Agricultural Center, Baton Rouge, LA 70803, USA for hiring a worker, $C^{w}=10$ (dollar/person/truck-load). The unit transportation cost for moving residue $k$ from the hub in zip code $z$ to the reactor be $\mathbf{C}^{\mathrm{t}}=\left[C_{z k}^{t}\right]=\left[\begin{array}{lll}3.3 & 4.1 & 3.7 \\ 3.3 & 3.3 & 4.0 \\ 4.7 & 4.7 & 3.8 \\ 4.2 & 4.2 & 3.1\end{array}\right]$ (dollar/truck-load/mile). The feedstock cost of residue $k$ supplied from the hub in zip code $z$ to the reactor is $\mathbf{C}^{r}=\left[C_{z k}^{r}\right]$ $=\left[\begin{array}{lll}100 & 104 & 122 \\ 139 & 120 & 146 \\ 141 & 113 & 109 \\ 144 & 140 & 113\end{array}\right]$ (dollar/truck-load). The unit cost of pipeline from the reactor $n$ to condenser $m, \mathbf{C}^{p}=\left[C_{n m}^{p}\right]=$ $\left[\begin{array}{ll}3.39 & 4.23 \\ 3.50 & 3.95\end{array}\right]$ (dollar/mile). The unit transportation cost for liquefied carbon product from condenser $m$ to delivery point $l, \mathbf{C}^{d}=\left[C_{m l}^{d}\right]=\left[\begin{array}{lll}3.70 & 4.17 & 4.83 \\ 4.66 & 4.10 & 3.57\end{array}\right]$ (dollar/truck-load/mile). The pipeline cost between reactors is $C_{1,2}^{b}=3.76$ (dollar/ mile). The demand of residues $k$ at the $n$th reactor is given as $\mathbf{D}=\left[D_{k n}\right]=\left[\begin{array}{ll}6 & 8 \\ 6 & 8 \\ 7 & 6\end{array}\right]$ (truck-load) and the unit workforce requirements for collecting residue $k, \mathbf{w}=\left[w_{k}\right]=\left[\begin{array}{l}3 \\ 5 \\ 4\end{array}\right]$ (persons/truck-load). Total workforce availability at the $n$th reactor, $\mathbf{W}=\left[W_{n}\right]=\left[\begin{array}{l}96 \\ 96\end{array}\right]$ (persons). Percent of residue to be unaccounted for due to natural loss or unavailability, $\beta=$ 0.05 .

The coordinate of the hub in zip code $z,\left[\left(x_{1}^{h}, y_{1}^{h}\right),\left(x_{2}^{h}, y_{2}^{h}\right.\right.$ ), $\left.\left(x_{3}^{h}, y_{3}^{h}\right),\left(x_{4}^{h}, y_{4}^{h}\right)\right]=[(56.88,16.22),(46.94,79.43),(1.19$, $31.12),(33.71,52.85)]$. The coordinate of the delivery points $\left.l,\left[\left(x_{1}^{p}, y_{1}^{p}\right),\left(x_{2}^{p}, y_{2}^{p}\right),\left(x_{3}^{p}, y_{3}^{p}\right)\right]\right]=[(116.56,165.41),(160.20$, 168.92), (126.30, 174.82)]. 
From the above data, the allocation sub-problem can be written in the form of (4). Applying one iteration of Algorithm C (ASA) yields:

Step C-1: Let $\overline{\mathbf{r}}_{1}=\overline{\mathbf{r}}_{2}=\frac{1}{2} \sum_{z=1}^{3} \mathbf{h}_{z}=\left[\begin{array}{l}69.36 \\ 89.81\end{array}\right]$ and $\overline{\mathbf{s}}_{1}=\overline{\mathbf{s}}_{2}=$ $\overline{\mathbf{r}}_{1}+\frac{1}{3} \sum_{l=1}^{3} \mathbf{p}_{l}=\left[\begin{array}{l}203.71 \\ 259.52\end{array}\right]$ be the initial location vectors for the reactors and condensers. $T C_{\text {opt }}$ is set to $+\infty$.

Step C-2: Algorithm B (B\&B method) is applied to find the optimal allocation $\overline{\boldsymbol{\alpha}^{1}}=\left[\begin{array}{lll}3 & 0 & 0 \\ 2 & 4 & 3 \\ 0 & 0 & 0 \\ 1 & 2 & 4\end{array}\right], \overline{\boldsymbol{\alpha}^{2}}=\left[\begin{array}{lll}3 & 0 & 0 \\ 2 & 4 & 3 \\ 0 & 0 & 0 \\ 3 & 4 & 4\end{array}\right], \overline{\boldsymbol{\gamma}}=$ $\left[\begin{array}{ll}1 & 0 \\ 0 & 1\end{array}\right]$ and $\overline{\boldsymbol{\rho}}=\left[\begin{array}{lll}1 & 0 & 0 \\ 0 & 1 & 1\end{array}\right]$ based on $\overline{\mathbf{r}}$ and $\overline{\mathbf{s}}$. Obtain the objective value as $\overline{T C}=\$ 16,061.53$

Step C-3: Algorithm A yields the locations $\tilde{\mathbf{r}}_{1}=$ $\left(\begin{array}{l}40.49 \\ 63.83\end{array}\right), \tilde{\mathbf{r}}_{2}=\left[\begin{array}{l}33.71 \\ 52.85\end{array}\right], \tilde{\mathbf{s}}_{1}=\left[\begin{array}{l}115.77 \\ 160.17\end{array}\right], \tilde{\mathbf{s}}_{2}=\left[\begin{array}{l}115.72 \\ 160.13\end{array}\right]$ based on allocations $\overline{\boldsymbol{\alpha}^{n}}, \overline{\boldsymbol{\gamma}}$ and $\overline{\boldsymbol{\rho}}$. Hence, the incumbent total cost as $T C=\$ 10,787.69$.

Step C-4: Because $T C<T C_{\text {opt }}$, then $T C_{o p t}=\tilde{T C}=$ $\$ 10,787.69, \boldsymbol{\alpha}_{o p t}^{n}=\overline{\boldsymbol{\alpha}^{n}}$ for $n=1, \ldots, N, \gamma_{o p t}=\bar{\gamma}$ and $\boldsymbol{\rho}_{\text {opt }}=$ $\overline{\boldsymbol{\rho}} \mathbf{r}_{\text {opt }}=\tilde{\mathbf{r}}$ and $\mathbf{s}_{\text {opt }}=\tilde{\mathbf{s}}$.
Step C-5: Because $|\overline{T C}-\tilde{T C}|=5273.8>$ set precision $=$ 0.1 , then $\overline{\mathbf{r}}_{1}=\tilde{\mathbf{r}}_{1}=\left(\begin{array}{l}40.49 \\ 63.83\end{array}\right), \overline{\mathbf{r}}_{2}=\tilde{\mathbf{r}}_{2}=\left[\begin{array}{l}33.71 \\ 52.85\end{array}\right], \overline{\mathbf{s}}_{1}=\tilde{\mathbf{s}}_{1}$ $=\left[\begin{array}{l}115.77 \\ 160.17\end{array}\right], \overline{\mathbf{s}}_{1}=\tilde{\mathbf{s}}_{2}=\left[\begin{array}{l}115.72 \\ 160.13\end{array}\right]$ and go to Step C-2.

A complete iteration shows how the computational procedure works using Algorithm B and C. The final outcome of the solution and the suboptimal solutions are in the most recent values of the variables from the $|\overline{T C}-\tilde{T C}|<$ set precision and updates to $\boldsymbol{\alpha}_{o p t}^{n}=\bar{\sim}^{n}$ for $n=1, \ldots, N, \boldsymbol{\gamma}_{o p t}=\overline{\boldsymbol{\gamma}}$ and $\boldsymbol{\rho}_{\text {opt }}=\overline{\boldsymbol{\rho}} \mathbf{r}_{\text {opt }}=\tilde{\mathbf{r}}$ and $\mathbf{s}_{\text {opt }}=\tilde{\mathbf{s}}$.

We propose an algorithm for the MINLP in (1) by alternatively searching minima for two sets of decoupled subproblems. A small instance illustrates the algorithm step by step. We present more numerical examples in the next section to test the efficiency of the algorithm.

The original article has been corrected.

Publisher's Note Springer Nature remains neutral with regard to jurisdictional claims in published maps and institutional affiliations. 